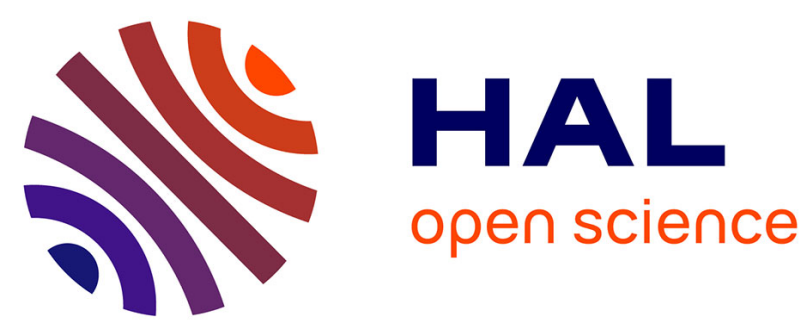

\title{
Large-scale vortex lattice emerging from collectively moving microtubules
}

\author{
Yutaka Sumino, Ken H. Nagai, Yuji Shitaka, Dan Tanaka, Kenichi \\ Yoshikawa, Hugues Chaté, Kazuhiro Oiwa
}

\section{To cite this version:}

Yutaka Sumino, Ken H. Nagai, Yuji Shitaka, Dan Tanaka, Kenichi Yoshikawa, et al.. Large-scale vortex lattice emerging from collectively moving microtubules. Nature, 2012, 483 (7390), pp.448-452. 10.1038 /nature10874 . cea-00881343

\section{HAL Id: cea-00881343 https://hal-cea.archives-ouvertes.fr/cea-00881343}

Submitted on 28 Sep 2020

HAL is a multi-disciplinary open access archive for the deposit and dissemination of scientific research documents, whether they are published or not. The documents may come from teaching and research institutions in France or abroad, or from public or private research centers.
L'archive ouverte pluridisciplinaire HAL, est destinée au dépôt et à la diffusion de documents scientifiques de niveau recherche, publiés ou non, émanant des établissements d'enseignement et de recherche français ou étrangers, des laboratoires publics ou privés. 


\title{
Large-scale vortex lattice emerging from collectively moving microtubules
}

\author{
Yutaka Sumino $^{1 *}$, Ken H. Nagai ${ }^{2 *}$, Yuji Shitaka ${ }^{3}$, Dan Tanaka ${ }^{4} \ddagger$, Kenichi Yoshikawa ${ }^{5}$, Hugues Chaté ${ }^{6}$ Kazuhiro Oiwa ${ }^{3,7}$
}

\begin{abstract}
Spontaneous collective motion, as in some flocks of bird and schools of fish, is an example of an emergent phenomenon. Such phenomena are at present of great interest ${ }^{1-5}$ and physicists have put forward a number of theoretical results that so far lack experimental verification ${ }^{6-8}$. In animal behaviour studies, large-scale data collection is now technologically possible, but data are still scarce and arise from observations rather than controlled experiments. Multicellular biological systems, such as bacterial colonies or tissues, ${ }^{9,10}$, allow more control, but may have many hidden variables and interactions, hindering proper tests of theoretical ideas. However, in systems on the subcellular scale such tests may be possible, particularly in in vitro experiments with only few purified components $\mathrm{s}^{11-13}$. Motility assays, in which protein filaments are driven by molecular motors grafted to a substrate in the presence of ATP, can show collective motion for high densities of motors and attached filaments. This was demonstrated recently for the actomyosin system ${ }^{14,15}$, but a complete understanding of the mechanisms at work is still lacking. Here we report experiments in which microtubules are propelled by surface-bound dyneins. In this system it is possible to study the local interaction: we find that colliding microtubules align with each other with high probability. At high densities, this alignment results in self-organization of the microtubules, which are on average $15 \mu \mathrm{m}$ long, into vortices with diameters of around $400 \mu \mathrm{m}$. Inside the vortices, the microtubules circulate both clockwise and anticlockwise. On longer timescales, the vortices form a lattice structure. The emergence of these structures, as verified by a mathematical model, is the result of the smooth, reptation-like motion of single microtubules in combination with local interactions (the nematic alignment due to collisions) - there is no need for long-range interactions. Apart from its potential relevance to cortical arrays in plant cells ${ }^{16,17}$ and other biological situations, our study provides evidence for the existence of previously unsuspected universality classes of collective motion phenomena.
\end{abstract}

Our experimental in vitro motility assay consisted of dynein motor proteins grafted to a glass surface at a density of 750-2,500 molecules per square micrometre and to fluorescently labelled, stabilized microtubules (details can be found in Supplementary Methods). We used inner-arm dynein subspecies c (dynein c) purified from Chlamydomonas flagella. This drove the microtubules, with their plus ends leading, at a mean velocity of $\sim 12 \mu \mathrm{m} \mathrm{s}^{-1}$ in vitro at $23^{\circ} \mathrm{C}$ in the presence of $1 \mathrm{mM}$ ATP, while rotating them around their longitudinal axis in a clockwise direction as viewed from their minus ends ${ }^{18,19}$. ('Plus' and 'minus' here refer to microtubule polarity.) When introduced in a flow cell, the microtubules did not move and bound to the dynein-c-coated glass surface in the absence of $\mathrm{ATP}^{18,19}$. On addition of $1 \mathrm{mM} \mathrm{Mg}-\mathrm{ATP}$, they started moving smoothly on the surface at a fairly constant speed. No preferred direction of isolated microtubule motion was observed. The smoothness and isotropy of motion reflect the fact that relatively many, randomly oriented, dyneins were attached at any time to a given microtubule.

Using microtubules at a concentration of $40 \mu \mathrm{g} \mathrm{ml}^{-1}$, which is a few times greater than in conventional assays ${ }^{18}$, we found the density of attached microtubules to be $0.05 \mu \mathrm{m}^{-2}$ (there were five microtubules, $15.6 \pm 7.3 \mu \mathrm{m}$ in length (mean \pm s.d., $n=257$ ), per $100 \mu \mathrm{m}^{2}$ ). On addition of ATP (at $0 \mathrm{~min}$ ), we observed many aligning collisions (discussed further below) that gradually led to streams along which dozens of microtubules moved in both directions. The size of a stream increased in time (by more than $10 \mu \mathrm{m}$ in width and more than $100 \mu \mathrm{m}$ in length) (12 min; Fig. 1a). The streams started to meander over the surface typically after $10 \mathrm{~min}$. At 10-20 min, some vortices appeared (17 min; Fig. 1a and Supplementary Movie 1) and eventually covered the flow cell almost entirely, except for the area less than $500 \mu \mathrm{m}$ away from the boundaries (Fig. 1b). The shape of the vortices gradually changed over time but their diameters were almost constant, at $400 \mu \mathrm{m}$ (major axis, $443 \pm 64 \mu \mathrm{m}$ (s.d., $n=40$ ); minor axis, $390 \pm 59 \mu \mathrm{m}$ (s.d., $n=40)$ ). At this late stage, the vortices showed a tendency to arrange their positions into a hexagonal lattice, although not perfectly. A given vortex consists of a sparse core and a dense peripheral annulus inside which microtubules move both clockwise and anticlockwise in small streams and slide past each other (Fig. 2 and Supplementary Movie 2). That is, the streams show nematic rather than polar order. Microtubules were never trapped in a vortex: they would circulate inside one vortex for some time before moving to a neighbouring one or travelling farther and starting to revolve around a more distant core.

As the first step in understanding vortex formation, we examined the interactions between microtubules. We decreased their density to $0.5-$ $1 \mu \mathrm{g} \mathrm{ml}^{-1}$ to focus on pair interactions between isolated microtubules. We examined 393 pair collision events. No significant interaction was detected until the pair collided, indicating that long-range, 'hydrodynamic' effects are negligible. On collision, strong interactions due to volume exclusion occurred overwhelmingly ( $80 \%$ of events), leading either to alignment or anti-alignment (70\%; Fig. 3a, b) or to the stoppage of one microtubule to prevent it from hitting the other (10\%; Fig. 3c). In the remaining fraction of events (20\%), the microtubules crossed each other with little effect on their trajectories (Fig. 3d, e; see also Supplementary Movie 3). In aligning and antialigning collisions, the trajectory of one microtubule undergoes a sharp turn (Fig. 3a, b) and alignment was observed to be near perfect. We believe that this is due to the leading end of the microtubule being relatively free from attached dyneins by comparison with rest of it: on meeting an obstacle, the tip of the microtubule can then bend more easily. Thus, the outgoing angle is near 0 or $\pi$ in aligning events, irrespective of the value of the incoming angle, $\phi$. In stoppage and crossing events, the orientations of both microtubules remain roughly unchanged. (During stoppage events, one microtubule simply 'waits'

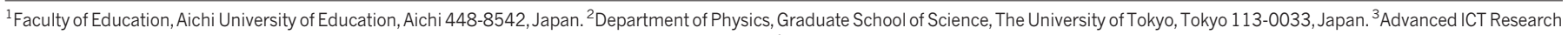

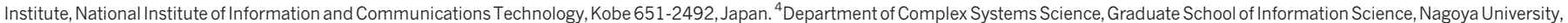

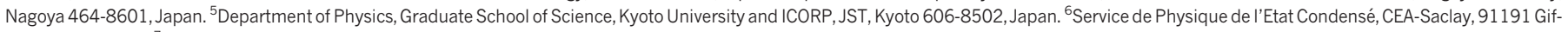
sur-Yvette, France. ${ }^{7}$ Graduate School of Life Science, University of Hyogo, Harima Science Park City, Hyogo 678-1297, Japan.

*These authors contributed equally to this work

tDeceased 
a

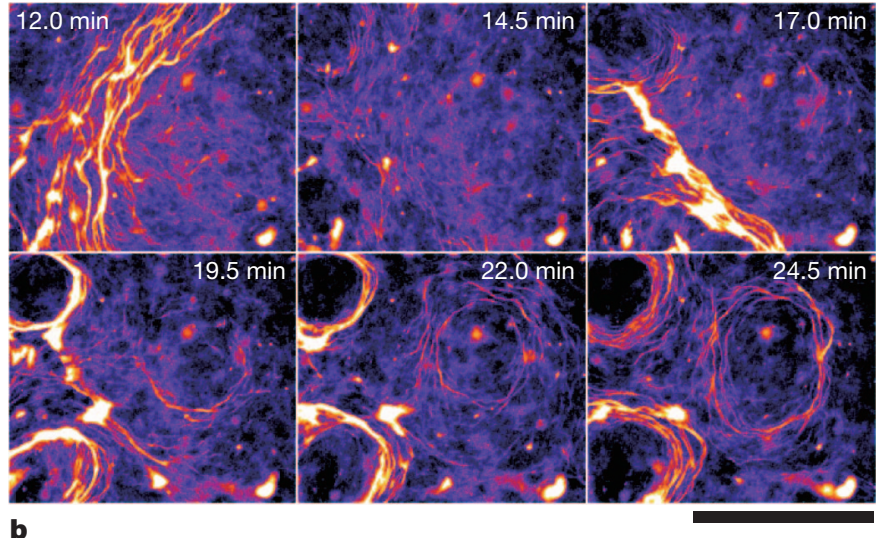

b

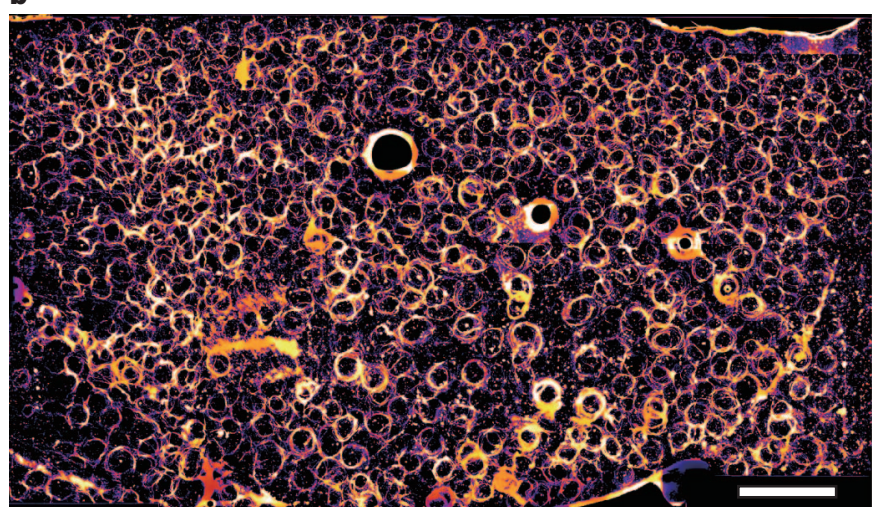

Figure $1 \mid$ Emergence of vortices of microtubules. False-colour images. a, Formation process of vortex pattern: 0 min corresponds to the time of injection of ATP. Streams appeared around $5 \mathrm{~min}$ after this injection. At $12 \mathrm{~min}$, streams started to meander. Vortices started to appear around $17 \mathrm{~min}$, when meandering streams contacted one another. Once vortices had formed, they grew steadily ( $22 \mathrm{~min}$ ). Scale bar, $500 \mu \mathrm{m}$. b. Large-scale lattice of vortices. Vortices can be observed everywhere on the surface of the flow cell. Three air bubbles in the flow cell can be seen distinctively owing to their greater size and thicker edges. Scale bar, $2 \mathrm{~mm}$.

until the other has passed (Fig. 3c).) Given that the outcome of each type of collision is well defined, we represented the complete collision statistics in terms of the probability of each collision type as a function of $\phi$ only (Fig. 3d). We observe an overall symmetry around $\phi=\pi / 2$, with alignment and anti-alignment occurring mainly for small $(\phi \approx 0)$ and large $(\phi \approx \pi)$ angles, respectively, whereas stoppage and crossing dominate for $\phi \approx \pi / 2$. Thus, we conclude that near-perfect nematic alignment is induced by direct collision.

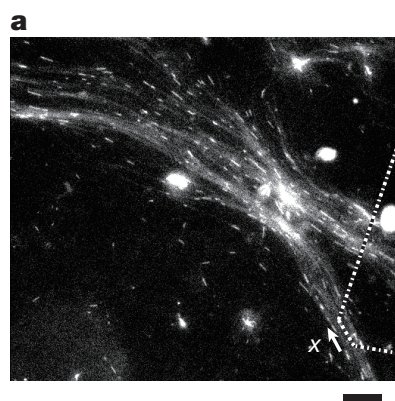

b

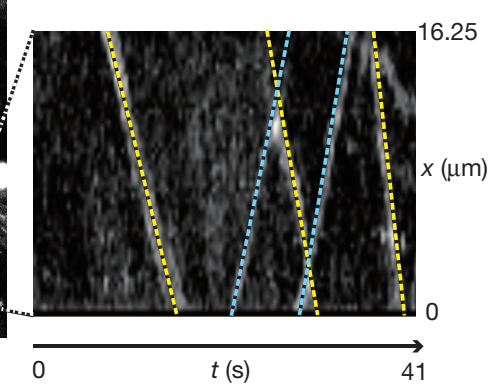

Figure $2 \mid$ Nematic order inside a vortex stream. In this experiment, the microtubules were all stained fluorescent but approximately $0.1 \%$ of microtubules were stained to be more fluorescent than the rest. a, Close-up of vortex. Scale bar, $20 \mu \mathrm{m}$. b. Spatiotemporal diagram of a magnified region of the vortex shown in a. Yellow lines indicate microtubules travelling from top to bottom, and blue lines indicate microtubules travelling from bottom to top. a

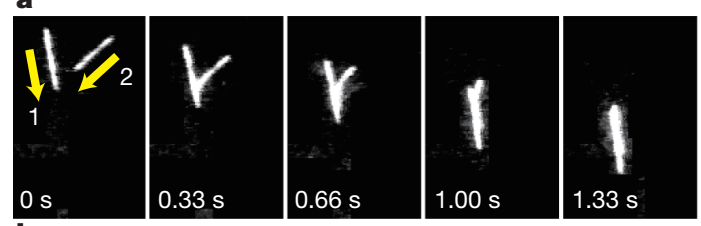

b
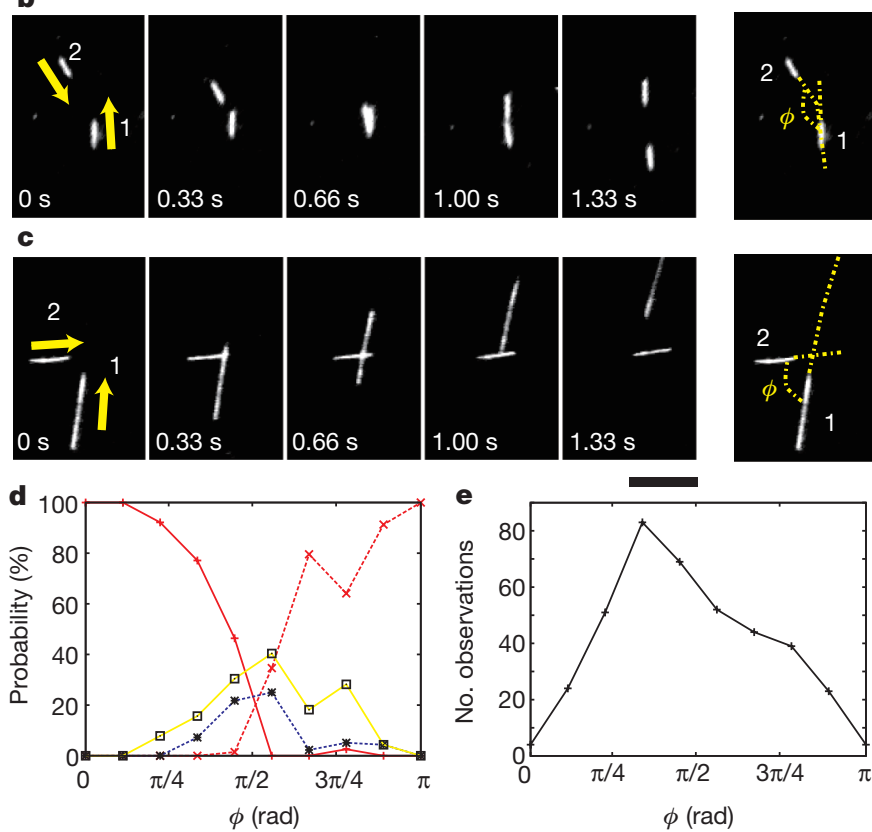

Figure 3 Collision between microtubules. a-c, Three different types of collision between two microtubules. In $\mathbf{a}$ and $\mathbf{b}$, microtubule 2 collides with the side of microtubule 1 and changes its direction of motion abruptly, leading to almost perfect alignment (a) or anti-alignment (b). In c, microtubule 2 stops until microtubule 1 has passed. Scale bar, $10 \mu \mathrm{m}$. d, Probability of each

interaction type as a function of $\phi$ (see right-most images in $\mathbf{a}-\mathbf{c}$ ). We analysed 393 events. Red lines represent alignment (solid) and anti-alignment (dotted) events; blue dotted lines represent stopping events; yellow solid lines represent crossing-over events. e, Total number of observations in each angular bin.

Constant-speed particles aligning nematically in the presence of noise have been studied recently in mathematical models of selfpropelled rods, which were shown to give rise to unexpected collective dynamics but not to vortical structures ${ }^{20}$. Our motility assay has properties not present in simple models of colliding rods. One such feature is the nature of the motion of isolated microtubules: as already mentioned, their reptation-like movement yields smooth but constantly turning trajectories, unlike both straight ballistic motion and the ragged behaviour of random walkers submitted to rotational diffusion, the individual dynamics adopted in rods models ${ }^{20-22}$. We analysed the trajectories of isolated microtubules for durations of up to $350 \mathrm{~s}$ (Fig. 4a, left). Here we further decreased their concentration, to $4.8 \mathrm{ng} \mathrm{ml}^{-1}$. The speed (tangential velocity) of the microtubules fluctuated in time, with a mean of $v_{0}=8.75 \mu \mathrm{m} \mathrm{s}^{-1}$. We also measured the direction of motion, $\theta$ (where $\theta=0$ and $\pi / 2$ correspond to the direction of the $x$ and $y$ axes, respectively), and the instantaneous angular velocity, and found them to have no significant correlation with the instantaneous speed. This allowed us to neglect the speed fluctuations and focus on $\theta$.

A closer look at a representative trajectory (Fig. 4a, right) reveals small-amplitude transverse oscillations, which might be caused by the rotation of the microtubule around its axis ${ }^{18}$. After an appropriate filtering, based on the Savitzky-Golay method, to eliminate these oscillations (Supplementary Methods), we obtained the variation of curvature, $\kappa=\mathrm{d} \theta / \mathrm{d} s$, as a function of distance, $s$, along the trajectory and calculated its autocorrelation (Fig. $4 \mathrm{~b}$ ). (With this definition, $\kappa$ can be positive 

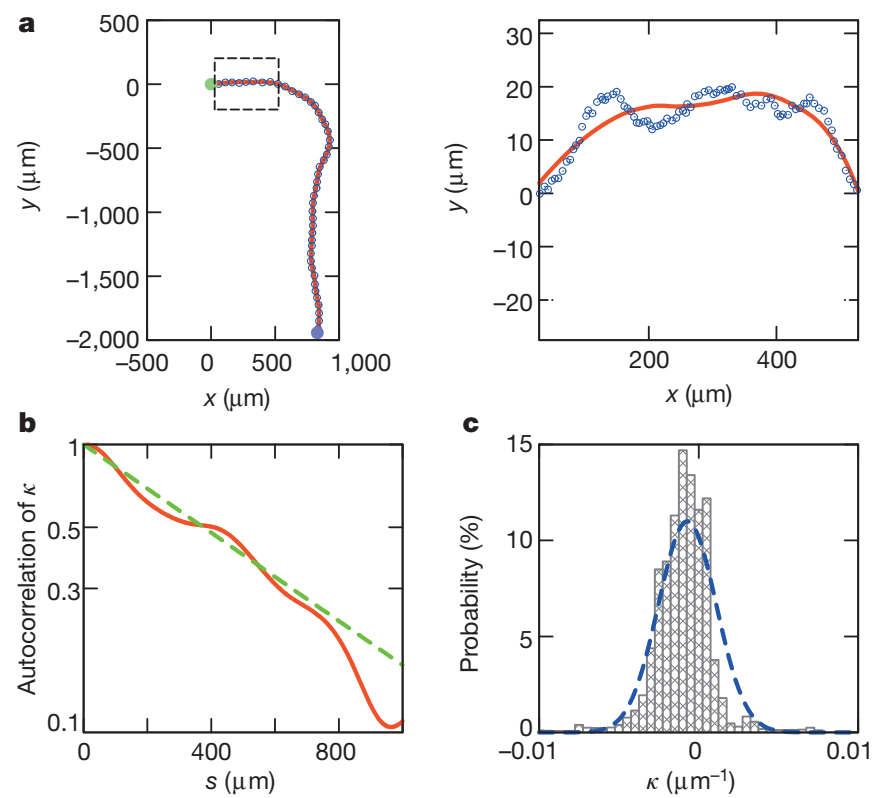

Figure $4 \mid$ Motion of isolated microtubules. a, Left: trace of an isolated microtubule. Right: enlarged image of boxed area on left. The green and blue filled circles correspond to the start and end points of the observation. The open blue dots represent the position of the microtubule, and the red solid line corresponds to the smoothed curve. $\mathbf{b}$, Autocorrelation of the trajectory curvature, $\kappa$, with respect to the distance along a trajectory, $s$ (red solid line). The green dotted line is an exponential fit with the correlation length $s_{0}=542 \pm 4 \mu \mathrm{m}$. c , Distribution of $\kappa$ values observed (bars) and Gaussian fit (dashed blue line): mean, $\kappa_{0}=-7.1 \times 10^{-4} \mu \mathrm{m}^{-1}$; s.d., $\sigma_{\kappa}=1.8 \times 10^{-3} \mu \mathrm{m}^{-1}$.

(anticlockwise motion) or negative (clockwise motion).) Fitting the result with the exponential function $\exp \left(-s / s_{0}\right)$, we found the persistent length, $s_{0}$, of curvature to be of the order of $500 \mu \mathrm{m}$. The distribution of $\kappa$ values observed is roughly Gaussian with mean $\kappa_{0}=-7.1 \times 10^{-4} \mu \mathrm{m}^{-1}$ and standard deviation $\sigma_{\kappa}=1.8 \times 10^{-3} \mu \mathrm{m}^{-1}$ (Fig. 4c). We note that these numbers correspond to length scales that are rather large and of the same order as the vortex diameter, and that the small negative value of $\kappa_{0}$ indicates a preference for clockwise turning in single-microtubule trajectories, which is probably a consequence of the clockwise rotation around of the microtubules around their axes while in motion ${ }^{23}$. A detailed molecular-level study should confirm this.

We constructed a mathematical model for the collective motion of microtubules in our experimental motility assay by incorporating the few ingredients uncovered in our analysis of the trajectories of single microtubules and of pair collisions. A deliberate choice of minimality was made, to stress that with only these very few features all observed collective phenomena are reproduced, and to allow for numerical simulations of millions of objects: for instance, the dynein motors driving the microtubules are not modelled explicitly; the hydrodynamic interactions induced by the fluid are neglected, in agreement with our observation that only direct contact modifies the microtubules' motion. The microtubules themselves are not represented as flexible polymers: here, in the spirit of ref. 6 , they are identical point particles moving at constant speed, $v_{0}$ (the speed fluctuations are also neglected). In direct agreement with the observations reported in Fig. 4, the free motion of particle $i$, with position $\mathbf{x}_{i}$ and orientation $\theta_{i}$, is a biased OrnsteinUhlenbeck process, $\omega_{i}(t)$, with correlation time $\tau=s_{0} / v_{0}$, acting on the particle's instantaneous angular velocity, $\mathrm{d} \theta_{i} / \mathrm{d} t$ :

$$
\begin{aligned}
\frac{\mathrm{d} \mathbf{x}_{i}}{\mathrm{~d} t} & =v_{0}\left(\mathbf{e}_{x} \cos \theta_{i}+\mathbf{e}_{y} \sin \theta_{i}\right) \\
\frac{\mathrm{d} \theta_{i}}{\mathrm{~d} t} & =\omega_{i}
\end{aligned}
$$

$$
\frac{\mathrm{d} \omega_{i}}{\mathrm{~d} t}=-\frac{1}{\tau}\left(\omega_{i}-\omega_{0}\right)+\xi(t)
$$

where $\mathbf{e}_{x}$ and $\mathbf{e}_{y}$ are respectively unit vectors in the $x$ and $y$ directions, $\xi(t)$ is a Gaussian white noise with zero mean and variance $\sigma^{2}=2 v_{0}^{2} \sigma_{\kappa}^{2} / \tau=2 v_{0}^{3} \sigma_{\kappa}^{2} / s_{0}$, and $\omega_{0}=v_{0} \kappa_{0}$ is the preferred non-zero angular velocity induced by the mean curvature, $\kappa_{0}$. This ensures that all correlation properties of the curvature measured in the experiment are reproduced (Supplementary Methods).

These equations must be complemented with an interaction term. Only the nematic alignment collisions are modelled (because stoppage and crossings do not substantially modify the microtubule orientations). As they were observed to induce much sharper turns in the trajectories (Fig. 3a, b) than the spontaneous curvature changes of free microtubules (Fig. 4a), these collisions are modelled as a nematic alignment term acting directly on the particle orientation $\theta_{i}$, that is, in equation (1), and not on the angular velocity, $\omega_{i}$. Following previous studies $^{6,20}$, we replaced explicit pair collisions by an effective alignment within an interaction range, $l$, which is naturally chosen to be the typical microtubule length. Equation (1) is thus replaced by

$$
\begin{aligned}
\frac{\mathrm{d} \mathbf{x}_{i}}{\mathrm{~d} t} & =v_{0}\left(\mathbf{e}_{x} \cos \theta_{i}+\mathbf{e}_{y} \sin \theta_{i}\right) \\
\frac{\mathrm{d} \theta_{i}}{\mathrm{~d} t} & =\omega_{i}+\frac{\alpha}{n_{i}(t)} \sum_{j \sim i} \sin \left(2\left(\theta_{j}-\theta_{i}\right)\right)
\end{aligned}
$$

where $\alpha$ is a parameter and the sum is over the $n_{i}(t)$ particles $j$ within distance $l$ of particle $i$ at time $t$.

Numerical simulations of equations (2) and (3) using experimentally obtained parameters (Supplementary Methods) show the emergence, from disordered initial conditions, of large-scale vortices with a diameter of the same order as those observed in experiments (Fig. 5a and Supplementary Movie 4).

Inside vortices, the simulated particles revolved both clockwise and anticlockwise, with the majority moving clockwise, as expected from the non-zero value of $\omega_{0}$ used. The number of vortices increased over time and saturated, leading eventually to a regular hexagonal lattice structure. As in our experimental observations, vortices did not form at low densities. To see this more clearly, we calculated $u^{2}(n)$, the variance of the local density in small boxes containing $n_{\mathrm{a}}$ particles on average, as a function of the rescaled average density, $\rho_{\mathrm{a}}$ (the actual density multiplied by $l^{2}$; Supplementary Methods), the other parameters being kept fixed (Fig. 5b). For $\rho_{\mathrm{a}}<0.4$, the system remains disordered and $u^{2}(n) / n_{\mathrm{a}}$ is almost constant, indicating the absence of density structures (Fig. 5b, right, images (i) and (ii)). However, $u^{2}(n) / n_{\mathrm{a}}$ increased with $\rho_{\mathrm{a}}$ for $\rho_{\mathrm{a}}>0.4$, indicating the appearance of a density pattern, which we checked visually to be a vortex lattice (Fig. 5b, right, images (iii) and (iv)). This result is consistent with our experimental observation that the vortex pattern disappears when the density of microtubules is halved (compare with Supplementary Fig. 1). We also performed numerical simulations in which we varied the rescaled correlation time, $\lambda=v_{0} \tau / l$ (Supplementary Methods), but kept all the other parameters fixed. Global nematic order without a density pattern was observed for $\lambda<60$, but the system showed vortex formation for $\lambda>60$ (Fig. $5 \mathrm{c}$ ). Thus, the large persistence length and time of single-microtubule motion is necessary for the emergence of the vortices. Further information on the phase diagram of our mathematical model can be found in Supplementary Methods and Supplementary Fig. 2.

The study of our mathematical model thus shows that smooth trajectories of single microtubules and their direct collisions inducing sharp bends and nematic alignment-both experimentally observedare sufficient, when associated with self-propulsion, to account for all observed collective properties. In particular, there is no theoretical reason to invoke, nor is experimental evidence of, long-range 'hydrodynamic' interactions or other 'collective effects' in our system. By 
a
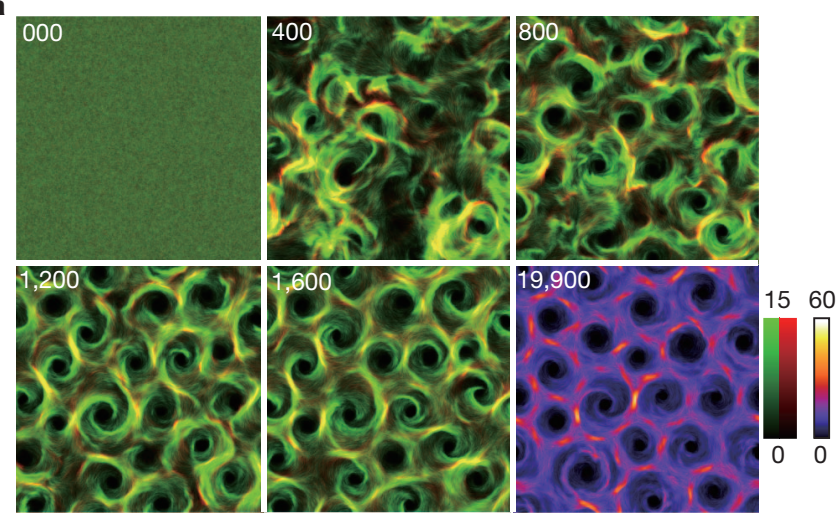

b
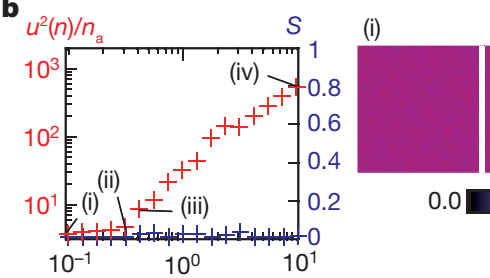

(ii)

(iii)
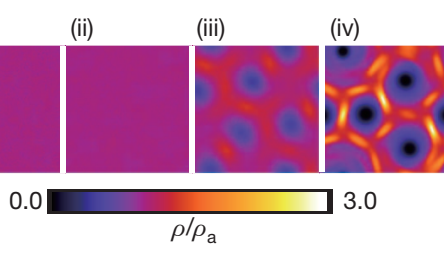

c

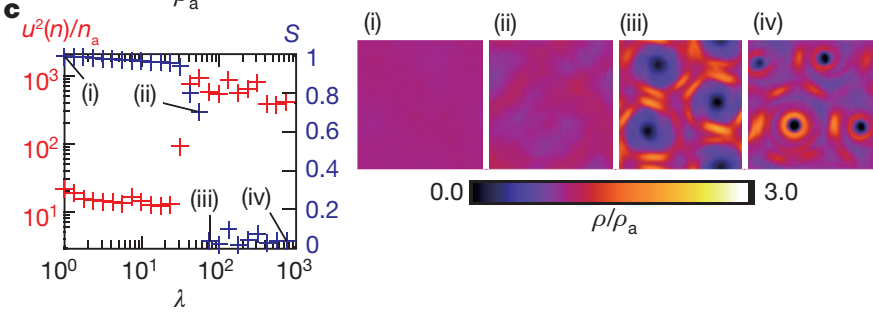

Figure 5 Collective dynamics of mathematical model of interacting, selfpropelled particles. See equations (2) and (3). a, Spontaneous formation of a vortex reproduced by our model. Each image is averaged for $\Delta T=10$, where $T$ is simulation time rescaled by $l / v_{0}$. The brightest area corresponds to a density of 15 particles per unit area. Green and red respectively correspond to the densities of particles moving clockwise $(\omega<0)$ and anticlockwise $(\omega>0)$. Yellow regions indicate areas with clockwise and anticlockwise motion in approximately equal proportions. The area of the figure is $512 \times 512$

simulation cells. The last snapshot, at $T=19,900$, shows the particle densities without differentiating between clockwise and anticlockwise motion, to make comparison with experimental results easy. b, Global nematic order parameter, $S$, and variance of the local density, $u^{2}(n)$ (calculated over boxes 4 simulation cells in linear size and $\Delta T=10)$, divided by the average density for the box, $n_{\mathrm{a}}$, plotted against $\rho_{\mathrm{a}}$. Corresponding density distributions are shown in images (i)-(iv) (each corresponds to a different value of $\rho_{\text {a }}$, as shown, and is a sum over 5,000 snapshots taken at intervals of $\Delta T=1$ in a $256 \times 256$ system). Here we set the rescaled correlation time, $\lambda=v_{0} \tau / l$, to $\lambda=100$. $\mathbf{c}$, Same as $\mathbf{b}$, but plotted against $\lambda$ with $\rho_{\mathrm{a}}=10$. The density distributions correspond to different values of $\lambda$, as shown.

contrast, such interactions were relied on to explain some of the collective phenomena observed in the actomyosin system ${ }^{14}$. We believe that our observations are a consequence of using dynein $c$ motors; in fact, when kinesin was used instead of dynein, microtubules mostly crossed each other and vortex formation could not be observed (Supplementary Fig. 3). With dynein, collisions are frequent enough to induce large-scale order. We suppose that this is due to the mechanical properties of dynein molecules and the asymmetry of their molecular structure. The formation of vortices arises from the smoothness and the curvature persistence of trajectories of single microtubules, both of which properties are, we believe, the consequence of having a relatively large number of motors attached at any time (we estimate it to be of the order of 100).

The vortices observed in our motility assay possess unique features: their large scale (relative to the length of microtubules), the irrelevance of hydrodynamic interactions and the dominantly nematic order inside them distinguish them from the polar, small-scale vortices formed by swimming sperm cells confined in quasi-two-dimensional conditions ${ }^{24}$. Their stability in time, and their well-defined diameters also distinguish them from the unstable 'swirling patterns' reported in the actomyosin assay ${ }^{14}$. From a theoretical viewpoint, we are not aware of other models showing a large-scale vortex pattern of the type found here. In our model, the crucial feature introduced is the combination of smooth, reptation-like motion and sharp nematic alignment. This combination can be seen as the signature of a new class in the emerging landscape of universality classes of collective motion phenomena.

Returning to the biological relevance of our findings, we believe that they stress further the message, in various in vivo situations and in particular in plant cell cortical arrays ${ }^{16,17,25,26}$, that simple physical collisions can induce the reorientation of microtubules, creating large-scale structures even in the absence, here, of crosslinkers ${ }^{27,28}$ Furthermore, there is a striking analogy between our analysis of microtubule collisions and that performed in plant cell cortex ${ }^{17,26,29}$, although there is only treadmilling and no actual displacement of microtubules in the cortex. As shown in Fig. 3d, in our system the probability of aligning collisions is high at small incoming angles $(\phi=0 \bmod \pi)$ and decreases monotonically from 0 to $\pi / 2$, as for 'zippering' (positive-end entrainment) of cortical microtubules.

Well-controlled in vitro experiments of the type reported here present crucial advantages due to the simplicity of the objects involved and the relative ease of collecting large amounts of data: not only can theoretical ideas be tested, but such experiments can reveal the importance of mechanisms previously ignored, and allow for quantitative analysis of features also present in vivo. They are and will remain invaluable tools for our understanding of active matter and biological organization processes.

\section{METHODS SUMMARY}

We prepared inner-arm dynein and tubulin using standardized protocols ${ }^{19}$. Cy3labelled microtubules were polymerized from a mixture of $\mathrm{Cy} 3$-tubulin and nonfluorescent tubulin and were stabilized using Taxol. Fractionated dynein $\mathrm{c}$ was diluted at various concentrations $\left(27-90 \mu \mathrm{g} \mathrm{ml}^{-1}\right)$ in buffer solution containing $30 \mathrm{mM}$ HEPES/KOH (pH 7.4), $5 \mathrm{mM} \mathrm{MgSO}_{4}, 1 \mathrm{mM}$ DTT, $1 \mathrm{mM} \mathrm{EGTA}$ and $1 \mathrm{mg}$ $\mathrm{ml}^{-1}$ bovine serum albumin. The flow cell was flushed with the diluted dynein solution, incubated for $5 \mathrm{~min}$ and then washed using the buffer solution. Solution containing microtubules as well as $1 \mathrm{mM}$ ATP and $1 \mathrm{mM}$ DTT was introduced into the flow cell. Microtubules were visualized on an epifluorescence microscope. The vortex pattern, binary collisions and individual trajectories were observed with solutions of microtubules with respective concentrations of $40 \mu \mathrm{g} \mathrm{ml}^{-1}$, $0.5-1 \mu \mathrm{g} \mathrm{ml}^{-1}$ and $4.8 \mathrm{ng} \mathrm{ml}^{-1}$. For single-trajectory analysis, the position of a microtubule was measured every $\Delta t=0.68 \mathrm{~s}$.

\section{Received 14 October 2011; accepted 17 January 2012.}

1. Ballerini, M. et al. Interaction ruling animal collective behavior depends on topological rather than metric distance: evidence from a field study. Proc. Natl Acad. Sci. USA 105, 1232-1237 (2008).

2. Buhl, J. et al. From disorder to order in marching locusts. Science $\mathbf{3 1 2}, \mathbf{1 4 0 2 - 1 4 0 6}$ (2006).

3. Katz, Y., Tunstrøm, K., loannou, C. C., Huepe, C. \& Couzin, I. D. Inferring the structure and dynamics of interactions in schooling fish. Proc. Natl Acad. Sci. USA 108, 18720-18725 (2011).

4. Lukeman, R., Li, Y.-X. \& Edelstein-Keshet, L. Inferring individual rules from collective behavior. Proc. Natl Acad. Sci. USA 107, 12576-12580 (2010).

5. Sumpter, D. J. T. Collective Animal Behavior (Princeton Univ. Press, 2010).

6. Vicsek, T., Czirók, A., Ben-Jacob, E., Cohen, I. \& Shochet, O. Novel type of phase transition in a system of self-driven particles. Phys. Rev. Lett. 75, 1226-1229 (1995).

7. Toner, J., Tu, Y. \& Ramaswamy, S. Hydrodynamics and phases of flocks. Ann. Phys 318, 170-244 (2005)

8. Ramaswamy, S. The mechanics and statistics of active matter. Annu. Rev. Condens. Mat. Phys. 1, 323-345 (2010).

9. Zhang, H. P., Be'er, A., Florin, E.-L. \& Swinney, H. L. Collective motion and density fluctuations in bacterial colonies. Proc. Natl Acad. Sci. USA 107, 13626-13630 (2010).

10. Poujade, M. et al. Collective migration of an epithelial monolayer in response to a model wound. Proc. Natl Acad. Sci. USA 104, 15988-15993 (2007).

11. Nédélec, F. J., Surrey, T., Maggs, A. C. \& Leibler, S. Self-organization of microtubules and motors. Nature 389, 305-308 (1997). 
12. Surrey, T., Nédélec, F., Leibler, S. \& Karsenti, E. Physical properties determining self-organization of motors and microtubules. Science 292, 1167-1171 (2001).

13. Loose, M., Fischer-Friedrich, E., Ries, J., Kruse, K. \& Schwille, P. Spatial regulators for bacterial cell division self-organize into surface waves in vitro. Science $\mathbf{3 2 0}$, 789-792 (2008).

14. Schaller, V., Weber, C., Semmrich, C., Frey, E. \& Bausch, A. R. Polar patterns of driven filaments. Nature 467, 73-77 (2010).

15. Butt, T. et al. Myosin motors drive long range alignment of actin filaments. J. Biol. Chem. 285, 4964-4974 (2010).

16. Yuan, M., Shaw, P. J., Warn, R. M. \& Lloyd, C. W. Dynamic reorientation of cortical microtubules, from transverse to longitudinal, in living plant cells. Proc. Natl Acad. Sci. USA 91, 6050-6053 (1994).

17. Wasteneys, G. O.\& Ambrose, J. C. Spatial organization of plant cortical microtubules: close encounters of the 2D kind. Trends Cell Biol. 19, 62-71 (2009)

18. Kagami, O. \& Kamiya, R. Translocation and rotation of microtubules caused by multiple species of Chlamydomonas inner-arm dynein. J. Cell Sci. 103, 653-664 (1992).

19. Sakakibara, H., Kojima, H., Sakai, Y., Katayama, E. \& Oiwa, K. Inner-arm dynein c of Chlamydomonas flagella is a single-headed processive motor. Nature $\mathbf{4 0 0}$, 586-590 (1999)

20. Ginelli, F., Peruani, F., Bär, M. \& Chaté, H. Large-scale collective properties of selfpropelled rods. Phys. Rev. Lett. 104, 184502 (2010).

21. Kudrolli, A., Lumay, G., Volfson, D. \& Tsimring, L. S. Swarming and swirling in selfpropelled polar granular rods. Phys. Rev. Lett. 100, 058001 (2008).

22. Peruani, F., Deutsch, A. \& Bär, M. Nonequilibrium clustering of self-propelled rods. Phys. Rev. E 74, 030904(R) (2006).

23. Kikushima, K. \& Kamiya, R. Clockwise translocation of microtubules by flagellar inner-arm dyneins. Biophys. J. 94, 4014-4019 (2008).

24. Riedel, I. H., Kruse, K. \& Howard, J. A self-organized vortex array of hydrodynamically entrained sperm cells. Science 309, 300-303 (2005)

25. Tindemans, S. H., Hawkins, R. J. \& Mulder, B. M. Survival of the aligned: ordering of the plant cortical microtubule array. Phys. Rev. Lett. 104, 058103 (2010).
26. Allard, J. F., Ambrose, J. C., Wasteneys, G. O. \& Cytrynbaum, E. N. A mechanochemical model explains interactions between cortical microtubules in plants. Biophys. J. 99, 1082-1090 (2010).

27. Hess, H. et al. Molecular self-assembly of "nanowires" and "nanospools" using active transport. Nano Lett. 5, 629-633 (2005).

28. Schaller, V., Weber, C., Hammerich, B., Frey, E. \& Bausch, A. R. Frozen steady states in active systems. Proc. Natl. Acad. Sci. USA 108, 19183-19188 (2011).

29. Dixit, R. \& Cyr, R. Encounters between dynamic cortical microtubules promote ordering of the cortical array through angle-dependent modifications of microtubule behavior. Plant Cell 16, 3274-3284 (2004).

Supplementary Information is linked to the online version of the paper at www.nature.com/nature.

Acknowledgements We thank H. Sakakibara, Y. Uchida and R. Nakamori for the preparation of the material and observations. They as well as $\mathrm{H}$. Kitahata are also thanked for discussions. Y. Sumino is supported by a Grant-in-Aid for Research Activity Start-up (no. 23840019), Grant-in-Aid for Scientific Research (B) (no. 21340023) and Grant-in-Aid for Scientific Research (A) (no. 20244067). K.H.N. would like to acknowledge the support of a fellowship from the JSPS (no. 23-1819).

Author Contributions K.O. discovered the phenomenon of vortex lattice formation described here and conceived and performed the experiments with Y. Shitaka. Y. Sumino, K.H.N., D.T. and H.C. conceived and designed the simulations. Y. Sumino, K.H.N. and H.C. performed and analysed the simulations. In vitro motility assays were carried out under the directions of K.O. Y. Sumino, K.H.N., Y. Shitaka, H.C., K.Y. and K.O. interpreted the data. Y. Sumino, K.H.N., H.C. and K.O. wrote the paper.

Author Information Reprints and permissions information is available at www.nature.com/reprints. The authors declare no competing financial interests. Readers are welcome to comment on the online version of this article at www.nature.com/nature. Correspondence and requests for materials should be addressed to K.O. (oiwa@nict.go.jp). 